\title{
Non-muscarinic Therapeutic Targets for Acute Organophosphorus Poisoning
}

\author{
Christopher Rosenbaum • Steven B. Bird
}

Published online: 8 June 2010

(C) American College of Medical Toxicology 2010

\begin{abstract}
Organophosphorus (OP) pesticides are a broad class of acetylcholinesterase inhibitors that are responsible for tremendous morbidity and mortality worldwide, contributing to an estimated 300,000 deaths annually. Current pharmacotherapy for acute OP poisoning includes the use of atropine, an oxime, and benzodiazepines. However, even with such therapy, the mortality from these agents is as high as $40 \%$. It is increasingly recognized that not all OPs are the same. Significant differences exist in their toxicity, lipophilicity, and response to oxime therapy. Other nonmuscarinic effects of OP pesticides exist, such as acute and chronic neuromuscular junction failure and central respiratory failure. In part because most of the mortality from these chemicals takes place in the developing world, little National Institutes of Health (NIH) research has been directed towards these agents. However, the similar mechanism of action of OP pesticides and the military nerve agents, along with increasing concerns about chemical terrorism has lead to the formation of the NIH Countermeasures Against Chemical Threats (CounterACT) Program. As part of the CounterACT Program, the $\mathrm{NIH}$ has recently designated six OP pesticides as "threat agents". This concept paper describes some of the knowledge gaps related to non-muscarinic effects of OP pesticides and highlights needed areas of further research. Leveraging the current NIH interest in these chemicals to medical necessities in the developing world offers the possibility of delivering new therapeutics where they are needed on a daily basis.
\end{abstract}

C. Rosenbaum $\cdot$ S. B. Bird $(\bowtie)$

Department of Emergency Medicine,

Division of Medical Toxicology,

University of Massachusetts Medical School,

Worcester, MA, USA

e-mail: steven.bird@umassmemorial.org
Keywords Organophosphorus · Nicotinic $\cdot$ Muscarinic . Poisoning

\section{Introduction}

Acute OP poisoning causes tremendous morbidity and mortality worldwide, and these toxins represent a class of terrorist "threat agents" against civilian populations. In part because of the similar mechanism of action to military nerve agents, most research into acute OP poisoning has focused on the muscarinic and CNS seizure effects of acetylcholinesterase (AChE) inhibition. Despite such ongoing research for more than 40 years, the mortality of acute OP poisoning continues to be as high as $40 \%$ [1-3], with no new therapies introduced in 30 years.

The acute toxicity of OPs is primarily due to inhibition of acetylcholinesterase [4, 5], leading to excess acetylcholine at synapses with the resultant cholinergic toxicity and muscle weakness. Current therapy for OP poisoning requires resuscitation with the use of oxygen and atropine, followed by administration of an oxime (such as pralidoxime (2-PAM)) to reactivate AChE, plus benzodiazepines to mitigate neurological impairment [6-10]. However, 2-PAM has a limited window of effectiveness and cannot regenerate functional $\mathrm{AChE}$ once the alkyl chains of the OP are removed by aging. Furthermore, a recent randomized controlled trial of 2PAM showed a trend toward harm with 2-PAM when compared to saline placebo [11]. Efficacy of current antidotes is therefore severely limited and new approaches to treatment for OP poisoning, such as pharmacologically targeting the NMJ, could lead to improved outcomes.

OP poisoning leads to three main effects, each with a different time of onset: (1) an acute cholinergic crisis, (2) 
OP-induced delayed neuropathy (OPIDN), and (3) intermediate syndrome (IMS). The acute cholinergic syndrome gives rise to muscarinic, nicotinic, and central effects, due to an accumulation of ACh. OPIDN is a rare polyneuropathy that begins roughly $1-3$ weeks after an exposure. IMS, however, is a common complication that remains a major contributor to the high morbidity and mortality in OP poisoning [12]. IMS was first described as paralysis occurring 1-4 days after resolution of the acute cholinergic syndrome [13]. Recent work suggests that IMS occurs much sooner [14-16]. Severe cases of IMS are associated with respiratory muscle weakness that requires prolonged mechanical ventilation.

The pathophysiology of IMS is not clearly understood $[15,17-24]$ but is generally believed to result from a persistent excess of ACh at the NMJ $[15,16,20,22,23,25-$ 27]. Recognition of the IMS, first described over 20 years ago, [28] has not led to a proliferation of inquiry as to the NMJ or nicotinic effects of OP pesticides. In fact, not until 2008 was a prospective trial of IMS occurrence and detection in humans published [29-31]. The occurrence and cause of NMJ failure after OP pesticide poisoning remains underappreciated and understudied. Therefore, interventions targeting the NMJ could yield tremendous dividends in mitigating the morbidity and mortality from OP poisoning.

Future studies directed towards understanding which of the adverse effects of OP pesticides are due entirely to AChE inhibition (including both muscarinic and nicotinic effects) and which are other direct effects of the pesticide are needed. For example, at very high concentrations, dimethoate causes death via cardiovascular collapse about 10 to $20 \mathrm{~h}$ after ingestion despite maximal medical therapy that leads to death at a mean of $24 \mathrm{~h}$ after poisoning [3].

\section{All OP Pesticides Are Not the Same}

While OP pesticides all inhibit AChE, this diverse group of toxins often has unique characteristics in acute poisonings due to their individual chemical properties (Table 1). The NIH's CounterACT Program has identified six OP pesticides that they consider high priority threat agents of opportunity, and the chemical structures are shown in Fig. 1. Thus, the clinical scenario and cause of death vary according to the OP pesticide [32]. For instance, death due to dichlorvos poisoning occurs very rapidly, often before reaching medical care, while dimethoate toxicity takes several hours to develop. Regardless of the pesticide, the consequences of progressive muscle and respiratory weakness often dominate the early clinical picture.

Another underappreciated consequence of the unique poisoning characteristics of OP pesticides is the variable response of pesticides to treatment with 2-PAM. Although 2-PAM is routinely used in most of the world (including the
USA), what has recently become clear is that some pesticides (such as dimethoate) do not respond to 2-PAM therapy and a "one-size fits all" approach to 2-PAM therapy may not be warranted. Therefore, new methods of treatment that are independent of 2-PAM's mechanism of action are needed urgently.

\section{Respiratory Complications After OP Poisoning}

Approximately $24 \%$ of OP poisoned patients require intubation. Of these, more than $50 \%$ die [33]. Most of these deaths result from acute respiratory failure, due to either central respiratory depression [8], respiratory muscle weakness, or direct pulmonary effects such as bronchospasm or bronchorrhea. Few studies exist, but recent laboratory evidence from isolated mouse diaphragms suggests that increased recovery of AChE activity leads to increased force of muscle contraction [33]. Thus, therapies directed towards improving neuromuscular transmission and/or improving recovery of $\mathrm{AChE}$ could prove beneficial.

\section{Acute Physiological and Neuromuscular Junction Effects of OPs Are Not Well Understood}

The neuromuscular junction consists of a presynaptic axon terminal and a postsynaptic muscle end plate. Within the presynaptic terminal are vesicles containing acetylcholine. Most of these vesicles are bound to the actin cytoskeleton by proteins called synapsins. When an action potential induces opening of calcium channels, increased intracellular calcium levels promote phosphorylation of synapsins. This phosphorylation results in release of the vesicles from their cytoskeletal sites [34].

After release from the cytoskeleton, vesicles become bound at the presynaptic membrane terminal in areas called active zones. This "docking" allows rapid exocytosis of the vesicles. Docking is mediated by proteins termed SNARES (soluble $N$-ethylmaleimide-sensitive-fusion-attachment protein receptors). SNARES attached to the terminal membrane form complexes with proteins located on the vesicle [35]. Proteins involved in SNARE complexes include vesicle-associated membrane protein, which is found on the vesicle surface, along with SNAP-25 (synaptosomalassociated protein) and syntaxin, proteins found at the terminal membrane [36]. Phosphorylation of docking proteins occurs in response to increased calcium levels. This induces SNARE complex formation, followed by exocytosis of the vesicle contents. The vesicle membrane becomes added to the terminal membrane. Vesicles are recycled when pits form in the terminal membrane and become coated with a protein called clathrin. These clathrin-coated pits then 
Table 1 Physico-chemical properties of the NIH-designated threat agents OPs, plus dimethoate for comparison

\begin{tabular}{|c|c|c|c|c|c|c|}
\hline OP pesticide & Chemistry & & & WHO toxicity class & Rat oral LD50 $(\mathrm{mg} / \mathrm{kg})^{\mathrm{a}}$ & Human mortality \\
\hline Chlorfenvinphos & Aromatic & Diethyl & Oxon & $\mathrm{Ib}$ & 15 & Unknown \\
\hline Chlorpyrifos & Aromatic & Diethyl & Thion & II & 220 & $10 \%$ \\
\hline Dicrotophos & Aliphatic & Dimethyl & Oxon & $\mathrm{Ib}$ & 20 & Unknown \\
\hline Dimethoate & Aliphatic & Dimethyl & Thion & II & 250 & $25 \%$ \\
\hline Disulfoton & Aliphatic & Diethyl & Thion & Ia & 10 & Unknown \\
\hline Methamidophos & Aliphatic & Dimethyl & Oxon & $\mathrm{Ib}$ & 20 & Unknown \\
\hline Parathion & Aromatic & Diethyl & Thion & Ia & 15 & $>20 \%$ \\
\hline
\end{tabular}

${ }^{a}$ LD50s represent means of values and best approximation from several studies

pinch off to form vesicles. Acetylcholine is then synthesized and repackaged into these vesicles. [37]

The postsynaptic membrane is heavily folded and invaginated. Acetylcholine receptors are found at the crests of the junctional folds, and voltage-sensitive $\mathrm{Na}^{+}$channels are concentrated within the folds. The acetylcholine receptors have an ideal binding constant to allow reversible binding of acetylcholine. When bound, ion channels within the receptor are opened with an influx of $\mathrm{Na}^{+}$, and there is a transient depolarization of the end-plate region. If this endplate potential is large enough, a muscle fiber action potential is generated, which leads to muscle contraction. Acetylcholine remaining in the synapse is rapidly degraded $\mathrm{AChE}$, and the muscle is allowed to repolarize.
Poisoning with OP pesticides causes numerous changes in the NMJ and myocytes, both due to (and independent of) AChE inhibition [19, 38-40]. There is currently a dearth of knowledge regarding the causes and mechanisms of NMJ failure during acute OP poisoning. Some authors have postulated that the NMJ failure is caused by overstimulation of acetylcholine receptors (AChR) due to the attendant high synaptic concentration of ACh [41] or by direct neurotoxicity [42]. The molecular mechanism of this is unknown, but may involve the desensitization of AChR [22]. It is also possible that the NMJ failure involves presynaptic dysfunction and vesicle depletion [43] or disruption of synaptic structure, such as nerve terminal retraction could also occur [44].
Fig. 1 Chemical structures of six threat agent OP pesticides, and dimethoate. a Chlorfenvinphos, b chlorpyrifos, c dicrotophos, d disulfoton, e methamidophos, $\mathbf{f}$ parathion, and $\mathbf{g}$ dimethoate<smiles>CCOP(=O)(OCC)OC(=CCl)c1ccc(Cl)cc1Cl</smiles><smiles>CCOP(=S)(OCC)Oc1nc(Cl)c(Cl)cc1Cl</smiles><smiles>COP(=O)(OC)O/C(C)=C/C(=O)N(C)C</smiles><smiles>CCCC[Se]P(=S)(OCC)OCC</smiles>

e<smiles>COP(N)(=O)SC</smiles><smiles>CCOP(=S)(OCC)Oc1ccc([N+](=O)[O-])cc1</smiles><smiles>CNC(=O)CSP(=S)(OC)OC</smiles> 


\section{Nicotinic Receptor Antagonists}

Nicotinic acetylcholine receptors (nAChR) can be broadly grouped into two classes-neuromuscular and neuronal. Neuronal $\mathrm{nAChR}$ are present in the brain, sympathetic and parasympathetic ganglia, and the adrenal medulla. The role of these receptors in the pathophysiology of acute OP poisoning is limited, and the prospects of pharmacologic ganglionic antagonism for OP poisoning treatment are guarded, mostly due to the significant hypotension caused by these medications [45].

Neuromuscular nAChR, however, are an attractive therapeutic target. Because acute OP pesticide poisoning leads to fasciculations and progressive weakness with overstimulation of these receptors, competitive antagonists of neuromuscular nAChR is a logical approach to mitigating the pesticide effects. Pharmacologic protection of the NMJ with nicotinic ACh receptor antagonists has only recently been theorized $[45,46]$.

Breningstall et al. published a fascinating case report of congenital NMJ end-plate AChE deficiency treated with intermittent intramuscular pancuronium [47]. While detailed neurophysiologic studies were not performed, they demonstrated improved tidal volumes and forced vital capacity after pancuronium therapy, which persisted for months. Besser et al. have published three studies investigating the ability of pancuronium to effect improvement in compound muscle action potentials (CMAP) after OP poisoning. In one study, two patients with acute severe organophosphate intoxication showed single evoked CMAP with repetitive discharges and prominent decremental responses of CMAP supramaximal nerve stimulation. Following the injection of a small dose of pancuronium, improvement in these abnormalities occurred and persisted for several hours [48]. Unfortunately, all of these patients were given pancuronium several days after OP poisoning (except for one patient that received pancuronium within $5 \mathrm{~h}$ of poisoning).

The above findings support the proposition that nAChR antagonists could improve muscle strength after acute OP poisoning. Controlled animal studies examining functional neuromuscular response and NMJ architecture changes with and without nAChR therapy are needed to determine if this class of agents could serve as potential therapy for acute OP poisoning.

\section{Conclusions}

There are significant non-muscarinic effects of acute OP poisoning, including nicotinic stimulation, NMJ dysfunction, and neural toxicity. These other effects, particularly the NMJ, represent a robust therapeutic target to mitigating the morbidity and mortality of acute OP poisoning.
Research focused on decreasing NMJ dysfunction and respiratory failure are urgently needed.

Acknowledgments This work was supported, in part, by a grant from the National Institute of Environmental Health Science (R21ES14019) to SBB.

This manuscript responds, in part, to the NIH program announcement PA-10-019.

\section{References}

1. Eyer F, Meischner V, Kiderlen D et al (2003) Human parathion poisoning. A toxicokinetic analysis. Toxicol Rev 22:143-163

2. Zilker T, Hibler A (1996) Treatment of severe parathion poison: clinical aspects. In: Szinicz L, Eyer P, Klimmek R (eds) Role of oximes in the treatment of anticholinesterase agent poisoning. Spektrum, Akademischer, Heidelberg, pp 9-17

3. Eddleston M, Eyer P, Worek F et al (2005) Differences between organophosphorus insecticides in human self-poisoning: a prospective cohort study. Lancet 366:1452-1459

4. Sidell FR (1994) Clinical effects of organophosphorus cholinesterase inhibitors. J Appl Toxicol 14:111-113

5. Thiermann H, Szinicz L, Eyer P et al (2005) Correlation between red blood cell acetylcholinesterase activity and neuromuscular transmission in organophosphate poisoning. Chem Biol Interact 157-158:345-347

6. Eddleston M, Dawson A, Karalliedde L et al (2004) Early management after self-poisoning with an organophosphorus or carbamate pesticide - a treatment protocol for junior doctors. Crit Care 8:R391-397

7. Sivilotti ML, Bird SB, Lo JC et al (2006) Multiple centrally acting antidotes protect against severe organophosphate toxicity. Acad Emerg Med 13:359-364

8. Bird SB, Gaspari RJ, Dickson EW (2003) Early death due to severe organophosphate poisoning is a centrally mediated process. Acad Emerg Med 10:295-298

9. Eddleston M, Singh S, Buckley N (2005) Organophosphorus poisoning (acute). Clin Evid 9:1744-1755

10. Ballantyne B, Marrs TC (1992) Overview of the biological and clinical aspects of organophosphates and carbamates. Clinical and experimental toxicology of organophosphate and carbamates. Butterworth Heinemann, Oxford, pp 3-14

11. Eddleston M, Eyer P, Worek F et al (2009) Pralidoxime in acute organophosphorus insecticide poisoning - a randomised controlled trial. PLoS medicine 6:e1000104

12. Senanayake N, Karalliedde L (1987) Neurotoxic effects of organophosphorus insecticides. An intermediate syndrome. N Engl J Med 316:761-763

13. Senanayake N, Johnson MK (1982) Acute polyneuropathy after poisoning by a new organophosphate insecticide. The New England journal of medicine 306:155-157

14. Li Y, Yu X, Wang Z et al (2006) Gastric lavage in acute organophosphorus pesticide poisoning (GLAOP) - a randomised controlled trial of multiple vs. single gastric lavage in unselected acute organophosphorus pesticide poisoning. BMC emergency medicine 6:10

15. Avasthi G, Singh G (2000) Serial neuro-electrophysiological studies in acute organophosphate poisoning - correlation with clinical findings, serum cholinesterase levels and atropine dosages. J Assoc Physicians India 48:794-799

16. Jayawardane P, Senanayake N, Dawson A (2009) Electrophysiological correlates of intermediate syndrome following acute 
organophosphate poisoning. Clinical toxicology Philadelphia, $\mathrm{Pa}$ 47:193-205

17. Singh G, Sidhu UP, Mahajan R et al (2000) Phrenic nerve conduction studies in acute organophosphate poisoning. Muscle \& nerve 23:627-632

18. Sedgwick EM, Senanayake N (1997) Pathophysiology of the intermediate syndrome of organophosphorus poisoning. Journal of neurology, neurosurgery, and psychiatry 62:201-202

19. Karalliedde L, Henry JA (1993) Effects of organophosphates on skeletal muscle. Human \& experimental toxicology 12:289-296

20. De Bleecker J, Van den Neucker K, Colardyn F (1993) Intermediate syndrome in organophosphorus poisoning: a prospective study. Critical care medicine 21:1706-1711

21. Benson B, Tolo D, McIntire M (1992) Is intermediate syndrome in organophosphate poisoning the result of insufficient oxime therapy. J Toxicol Clin Toxicol 30:347-349

22. John M, Oommen A, Zachariah A (2003) Muscle injury in organophosphorous poisoning and its role in the development of intermediate syndrome. Neurotoxicology 24:43-53

23. Dandapani M, Zachariah A, Kavitha MR et al (2003) Oxidative damage in intermediate syndrome of acute organophosphorous poisoning. The Indian journal of medical research 117:253-259

24. Katz EJ, Cortes VI, Eldefrawi ME et al (1997) Chlorpyrifos, parathion, and their oxons bind to and desensitize a nicotinic acetylcholine receptor: relevance to their toxicities. Toxicology and applied pharmacology 146:227-236

25. De Bleecker J, Vogelaers D, Ceuterick C et al (1992) Intermediate syndrome due to prolonged parathion poisoning. Acta neurologica Scandinavica 86:421-424

26. De Bleecker J, Lison D, Van Den Abeele K et al (1994) Acute and subacute organophosphate poisoning in the rat. Neurotoxicology 15:341-348

27. De Bleecker JL (1995) The intermediate syndrome in organophosphate poisoning: an overview of experimental and clinical observations. J Toxicol Clin Toxicol 33:683-686

28. Jayawardane P, Dawson AH, Weerasinghe V et al (2008) The spectrum of intermediate syndrome following acute organophosphate poisoning: a prospective cohort study from Sri Lanka. PLoS medicine 5:e147

29. Pond W, Houpt K (1978) The pig as a model in biomedical research. Comstock, London

30. Larsen M, Rolin B (2004) Use of the Gottingen minipig as a model of diabetes, with special focus on type 1 diabetes research. ILAR Journal 45:303-313

31. Skytte C, Makin A (2008) Measurement of cardiovascular effects in minipigs using telemetry. J Pharmacol Toxicol 58:172

32. Thiermann H, Eyer P, Worek F et al (2005) Effects of oximes on muscle force and acetylcholinesterase activity in isolated mouse hemidiaphragms exposed to paraoxon. Toxicology 214:190-197

33. Eddleston M, Mohamed F, Davies JO et al (2006) Respiratory failure in acute organophosphorus pesticide self-poisoning. QJM 99:513-522

34. Schiavo G, Matteoli M, Montecucco C (2000) Neurotoxins affecting neuroexocytosis. Phys Rev 80:717-766

35. Sollner T, Whiteheart SW, Brunner M et al (1993) SNAP receptors implicated in vesicle targeting and fusion. Nature $362: 318-324$

36. Schwartz JH (1991) Principles of Neural Science 3 rd ed. Appleton and Lange, Norwalk

37. Lotti M (2001) Clinical toxicology of anticholinesterase agents in humans. Handbook of Pesticide Toxicology. Academic, San Diego, pp 1043-1085

38. Evron T, Geyer BC, Cherni I et al (2007) Plant-derived human acetylcholinesterase- $\mathrm{R}$ provides protection from lethal organophosphate poisoning and its chronic aftermath. Faseb J 21:2961-2969

39. Colombo A, Orsi F, Bonfanti P (2005) Exposure to the organophosphorus pesticide chlorpyrifos inhibits acetylcholinesterase activity and affects muscular integrity in Xenopus laevis larvae. Chemosphere 61:1665-1671

40. Wecker L, Mrak RE, Dettbarn WD (1986) Evidence of necrosis in human intercostal muscle following inhalation of an organophosphate insecticide. Fundam Appl Toxicol 6:172-174

41. Tuler SM, Bowen JM (1989) Toxic effects of organophosphates on nerve cell growth and ultrastructure in culture. J Toxicol Environ Health 27:209-223

42. Flynn CJ, Wecker L (1986) choline levels in brain. A noncholinergic component of organophosphate toxicity. Biochemical pharmacology 35:3115-3121

43. Worek F, Mast U, Kiderlen D et al (1999) Improved determination of acetylcholinesterase activity in human whole blood. Clin Chim Acta 288:73-90

44. Padmaja D, Mantha S (2002) Monitoring of Neuromuscular Juntion. Ind J Anaesth 46:279-288

45. Sheridan RD, Smith AP, Turner SR et al (2005) Nicotinic antagonists in the treatment of nerve agent intoxication. J R Soc Med 98:114-115

46. White SM, Palfreman TM (2005) Nicotinic antagonists and nerve gas poisoning. J R Soc Med 98:336

47. Breningstall GN, Kurachek SC, Fugate JH et al (1996) Treatment of congenital endplate acetylcholinesterase deficiency by neuromuscular blockade. J Child Neurol 11:345-346

48. Besser R, Vogt T, Gutmann L (1990) Pancuronium improves the neuromuscular transmission defect of human organophosphate intoxication. Neurology 40:1275-1277 